\title{
O Ensino Integrado de Gestão de Qualidade de Software e Engenharia de Software Aplicada como Modelo de Educação Interdisciplinar: Um Relato de Experiência no Ensino de Graduação
}

\author{
Yuska P. C. Aguiar ${ }^{1}$, Juliana Saraiva ${ }^{1}$ Jorge Dias $^{1}$, Yuri Malheiros ${ }^{1}$ \\ ${ }^{1}$ Departamento de Ciências Exatas - Universidade Federal da Paraíba (UFPB) \\ Rua da Mangueira, s/n, - Companhia de Tecidos, 58297-000 - Rio Tinto - PB - Brasil \\ \{yuska, julianajags, jorge, yuri\}@dcx.ufpb.br

\begin{abstract}
The teaching-learning process of disciplines related to software quality can be a difficult task because of the abstract nature of the concepts involved. This paper presents the experience of applying an interdisciplinary approach between the disciplines Software Quality Management (SQM) and Applied Software Engineering (ASE), where the processes, projects and products developed in ASE course were evaluated by students of SQM. 38 students were involved in this experience, evaluating 5 real projects. It was possible to observe that setting content was better understood by the students, the approval of the teaching method applied, and an improvement of students' background targeting the software industry.
\end{abstract}

Resumo. $O$ processo ensino-aprendizagem de disciplinas relacionadas à Qualidade de Software pode ser dificultado pela natureza abstrata dos conceitos ensinados. Este artigo apresenta o relato de experiência da aplicação de uma abordagem interdisciplinar entre as disciplinas de Gestão de Qualidade de Software (GQS) e Engenharia de Software Aplicada (ESA), onde os processos, projetos e produtos desenvolvidos na disciplina de ESA foram avaliados pelos alunos de GQS. Foram envolvidos 38 alunos avaliando 5 projetos reais. Com adoção desse modelo, foi possível observar uma melhor compreensão do conteúdo pelos alunos, a aprovação do método de ensino e o melhor preparo dos alunos para o mercado da indústria de software.

\section{Introdução}

A avaliação de qualidade de software é considerado um atributo crítico no processo de desenvolvimento e implantação de software. Consequentemente, o reconhecimento da importância de gerir a qualidade torna-se um ponto relevante a ser ensinado pelos alunos dos cursos relacionados com Tecnologia da Informação [Thompson and Edwards 2000]. Neste contexto, o processo ensino-aprendizagem é uma atividade que vem sendo estudada há anos e professores têm enfrentado o desafio de promover um ambiente de ensino-aprendizagem que forme o aluno sob uma perspectiva técnica, mas também humanística [Gnatz et al. 2003]. Isto é portanto, um cenário comum em disciplinas relacionadas ao ensino de Engenharia de Software e vários pesquisadores possuem trabalhos relacionados a este tema [Suryn 2007; Wermelinger et al. 2015]. 
$\mathrm{Na}$ ementa da Disciplina de Gestão da Qualidade de Software (GQS) os conceitos a serem abordados são teóricos e abstratos. A inexistência de atividades práticas que possibilitem tornar os conceitos mais concretos podem dificultar o aprendizado por parte dos alunos. No contexto da UFPB (Campus IV), foi possível observar a possibilidade de interdisciplinaridade entre esta disciplina e a disciplina de Engenharia de Software Aplicada (ESA). A sinergia existente entre as duas disciplinas, tanto em termos de conteúdo, quanto de metodologia de ensino-aprendizagem, permitiu a experiência de uma abordagem interdisciplinar envolvendo professores e alunos de ambas as disciplinas durante o semestre de 2014.1 na referida instituição. Portanto, este artigo consiste em um relato da experiência da integração entre as atividades de projeto prático interdisciplinar envolvendo os alunos das disciplinas de ESA e GQS. A Seção 2 descreve a experiência, enquanto a Seção 3 discute os resultados. Por fim, na Seção 4, as lições aprendidas e as considerações finais são expostas.

\section{Descrição da Experiência}

A experiência se deu nos cursos de Bacharelado em Sistemas de Informação e de Licenciatura em Ciência da Computação da Universidade Federal da Paraíba (UFPB), Campus IV, no Centro de Ciências Aplicadas e Educação (CCAE), situada na cidade de Rio Tinto/PB. Ela envolveu as disciplinas de GQS e ESA que são oferecidas para ambos os cursos no mesmo semestre, para alunos do sétimo e quinto períodos, respectivamente. Participaram da experiência os 2 professores das disciplinas supracitadas, além de 18 alunos regularmente matriculados na disciplina de GQS e 20 alunos regularmente matriculados na disciplina de ESA, totalizando assim 38 alunos. Segundo a ementa contida nos Projetos Pedagógicos dos Cursos (PPCs), a disciplina de ESA visa aplicar os conceitos teóricos de Engenharia de Software (ES), disciplina específica que a antecede, em projetos práticos. Por outro lado, a ementa da disciplina de GQS oferecida tem como objetivo apresentar aos alunos o histórico e o conceito de qualidade de software, assim como normas, modelos de maturidade e métricas de qualidade, técnicas de garantia de qualidade, testes de software e modelos de melhoria do processo.

Neste contexto, os assuntos mencionados anteriormente foram ministrados separadamente pelos respectivos professores das disciplinas. No entanto, a interdisciplinaridade aconteceu na execução de projetos das duas disciplinas, por parte dos alunos. Na disciplina de ESA, foi exigido dos alunos que aplicassem um Processo de Desenvolvimento de Software (PDS) para produção de um produto final - atendendo a uma demanda real, destinados à resolução de problemas não fictícios. Assim, os alunos vivenciaram na prática a construção de diferentes artefatos recomendados por esses modelos de processo. Como resultado, 5 softwares foram desenvolvidos. Já o projeto da disciplina de GQS abrangeu um total de seis roteiros (entregáveis), dos quais quatro guiavam os alunos na elaboração de um plano de avaliação de qualidade de software (PAQS), e dois consistiam na execução do PAQS - cuja construção foi gradativa ao longo do semestre. Portanto, o PAQS contempla um conjunto de itens de investigação definidos enquanto perguntas a serem respondidas pela equipe de desenvolvimento (a partir de entrevista ou aplicação de questionário); itens de verificação para os artefatos gerados; ou itens dependentes da execução do produto de software. A turma foi organizada em 6 "equipes de qualidade" (de 3 ou 4 alunos) a fim 
de relacionar com as 6 "equipes de desenvolvimento". Segue uma breve descrição dos roteiros:

Roteiro 01: Qualidade do processo de desenvolvimento de software - De posse da descrição do PDS, cada equipe de qualidade deveria: a) caracterizar o ciclo de vida do PDS; b) identificar se o processo prevê práticas de desenvolvimento centrado no usuário ou de estimulo ao design participativo; c) identificar, e representar visualmente, como o ciclo de vida esta organizado em termos estruturais; e por fim, d) elaborar um conjunto de pontos positivos e negativos que poderiam interferir na qualidade do processo ou do produto.

Roteiro 02: Fatores Humanos - Os alunos deveriam definir um conjunto de "itens de investigação" sobre fatores humanos a serem inserido no PAQS de acordo com as categorias de: Peopleware, Inteligência Emocional, Liderança, Motivação e Comunicação. Os itens foram acrescentados ao PAQS enquanto um conjunto de questionamentos, direcionados aos desenvolvedores, para investigar aspectos que poderiam ter interferido no seu comportando durante o projeto. A execução deste roteiro foi precedida por duas visitas técnicas realizadas em empresas de desenvolvimento de software na cidade de Recife/PE. A iniciativa de levar os alunos para um ambiente real de desenvolvimento de software teve como objetivo diminuir a distância entre indústria e academia, sendo a experiência fundamental para a realização do roteiro 02 .

Roteiro 03: Métricas de produto de software - As equipes de qualidade tiveram acesso a versão inicial dos produtos desenvolvidos em ESA. Em seguida os alunos foram instruídos a aplicar a abordagem GQM (Goal Question Metric) [Basili and Weiss, 1984] a fim de definir as métricas a serem mensuradas para o software em avaliação. Optou-se pelo GQM com objetivo de estimular os alunos a refletirem sobre: Objeto do estudo, Objetivo, Enfoque de qualidade, Ponto de vista, Contexto.

Roteiro 04: Validação e Verificação de software - Neste momento, de posse da especificação do sistema, os alunos realizaram duas atividades: (i) estudar a documentação com objetivo de encontrar problemas na especificação dos sistema; (ii) especificar casos de teste com base na descrição dos requisitos funcionais e não funcionais do sistema, assim como dos casos de uso definidos para o mesmo. Como resultado, os casos de teste foram inseridos no PAQS, mas a execução destes aconteceu apenas na Roteiro 06.

Roteiro 05: Adequação do PAQS - As equipes de qualidade revisitaram todo o PAQS a fim de priorizar quais aspectos seriam mantido para a avaliação final. Além disso, os alunos de GQS puderam identificar e definir quais dos "itens de investigação" seriam obtidos a partir de inspeções, de questionários ou entrevistas e quais dependiam da execução do sistema. Diante das decisões os documentos necessário foram elaborados (a exemplo dos questionários).

Roteiro 06: Execução do PAQS - Cada equipe de qualidade realizou a avaliação, de acordo com o PAQS definido por sua equipe, para um software desenvolvido na disciplina de ESA. Como houve desistência de uma equipe de desenvolvimento ao longo da disciplina, 2 equipes de qualidade avaliaram o mesmo produto de software. 
Na Figura 1 tem-se um resumo dos entregáveis exigidos na disciplina de ESA, assim como os roteiros realizados pelos alunos de $\mathrm{GQS}^{1}$. Os entregáveis e roteiros estão organizados ao longo de 4 meses, tempo que compõe o período letivo da execução das disciplinas na UFPB (2014.1). É perceptível à exigência dos entregáveis de forma incremental nas duas disciplinas, assim os alunos recebiam feedback dos professores para prosseguirem considerando os ajustes a serem feitos.

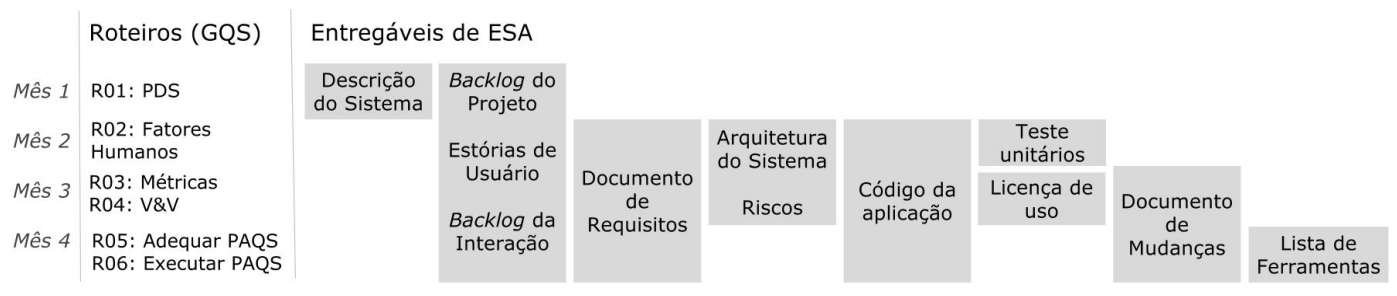

Figura 1. Relação temporal dos entregáveis de ESA e a execução dos roteiros de GQS.

\section{Discussão de Resultados}

Na tentativa de avaliar a abordagem metodológica utilizada na disciplina de GQS, duas perspectivas foram consideradas: (i) maturidade dos alunos em relação aos conceitos sobre Qualidade de Software; e (ii) avaliação dos alunos sobre a realização do projeto da disciplina. Para a primeira avaliação, realizou-se um fórum sobre qualidade de software com a participação de profissionais convidados. Os alunos foram estimulados a elaborar, previamente, um conjunto de até três perguntas sobre os temas abordados: "Barreiras e facilitadores para a implantação do processo de software" e "O que se espera de um profissional que atua na área de qualidade de software?". Ao todo foram enviadas 66 perguntas, das quais 32 foram definidas pelos professores como pertinentes para promover a discussão no fórum. Dentre os itens não selecionados, encontram-se perguntas muito semelhantes aquelas já contempladas na seleção. Seguem duas perguntas selecionadas para realização do fórum a fim de exemplificar o nível de maturidade dos alunos sobre Gestão da Qualidade de Software:

"Vemos que existem diversas barreiras para implementação de um processo de software, principalmente se um outro processo já vem sendo usado a tempos pela organização ou empresa. Sabendo disso, podemos falar que a maior barreira existente para essas mudanças seria o próprio homem? Ou seja o fator humano no desenvolvimento elou manutenção de software?";

"Hoje vemos que muitas empresas de tecnologia abrem e em pouco tempo fecham, muitas delas seus softwares são considerados de baixa qualidade, até mesmo por falta de planejamento. Minha dúvida é a seguinte, se está tão claro que a qualidade de software está ligada ao processo do mesmo por que as empresas não investem nessa área? Custo ou falta de informação?"

A segunda abordagem de avaliação foi realizada a partir da aplicação de questionário online ${ }^{2}$. O objetivo do questionário foi refinar a metodologia com turmas dos semestres seguintes. As perguntas podem ser organizadas em quatro categorias: (i) sobre as dificuldades encontradas na elaboração e execução do PAQS; (ii) sobre a

${ }^{1}$ http://tinyurl.com/qeobq9x

${ }^{2}$ http://goo.gl/forms/3jbqRYpaBd 
qualidade dos resultados alcançados pela equipe de qualidade; (iii) sobre a realização do projeto interdisciplinar, envolvendo as disciplinas de GQS e ESA; e (iv) sobre a disciplina, em uma perspectiva mais geral. As respostas foram fornecidas de forma espontânea, e dos 18 alunos que finalizaram o semestre, 17 responderam ao questionário. A seguir são apresentados e discutidos os resultados obtidos e as lições aprendidas com essa experiência de ensino.

\subsection{Dificuldades encontradas na elaboração e execução do PAQS}

A Tabela 1 apresenta, em resumo, as dificuldades encontradas pelos alunos nas execuções dos roteiros da disciplina de GQS. É possível observar que "identificar $e$ mensurar a qualidade do software" foi considerada como atividade mais difícil de ser executada. Esta situação pode ser explicada pelo fato de que mensurar a qualidade de algo intangível (como software) não é uma tarefa trivial. Se faz necessário que o engenheiro de software defina, dentre uma infinidade de possibilidade, um conjunto de objetivos pelos quais a qualidade deve ser mensurada. Portanto, é esperado que os alunos que estão em processo de formação e que, em sua maioria, têm pouca experiência prática, sintam dificuldade em executar esta atividade. Dois comentários podem explicitar esta realidade: "Foi uma atividade bastante complexa, na qual não tínhamos tanta experiência."; "Nunca ter feito isso antes. Mesmo com as aulas teóricas, a prática não era muito fácil, ficava na dúvida: por onde começar e como vou fazer".

Tabela 1. Dificuldades encontradas pelos alunos na execução dos roteiros

\begin{tabular}{|l|l|l|l|l|}
\hline Descrição das Dificuldades & $\mathbf{\%}$ & Descrição das Dificuldades & \% \\
\hline $\begin{array}{l}\text { Analisar o processo de desenvolvimento de } \\
\text { software adotado na disciplina de ESA }\end{array}$ & $\mathbf{2 5}$ & $\begin{array}{l}\text { Elaborar o questionário a ser respondido } \\
\text { pelos desenvolvedores do software avaliado }\end{array}$ & $\mathbf{2 5}$ \\
\hline $\begin{array}{l}\text { Identificar um conjunto de métricas para } \\
\text { mensurar a qualidade do software avaliado }\end{array}$ & $\mathbf{6 8 . 8}$ & $\begin{array}{l}\text { Elaborar parecer sobre a qualidade do } \\
\text { software avaliado }\end{array}$ & $\mathbf{0}$ \\
\hline $\begin{array}{l}\text { Especificar os casos de teste para o software } \\
\text { avaliado }\end{array}$ & $\mathbf{3 1 . 3}$ & Outros & $\mathbf{0}$ \\
\hline
\end{tabular}

Pelos percentuais obtidos, as três outras dificuldades encontradas podem ser classificadas como moderadas. Acreditamos que estes números podem ser explicados pelo fato da inexperiência dos alunos no processo de controlar e assegurar a qualidade do software. Todo processo de ensino tem uma curva de aprendizagem e à medida em que os alunos iam sendo apresentados às teorias e técnicas, logo em seguida, eles deveriam executar estas atividades.

\subsection{Qualidade dos resultados alcançados pela equipe de qualidade}

Os alunos realizaram uma auto-avaliação sobre os resultados que alcançaram enquanto equipe de qualidade em relação ao PAQS elaborado e ao diagnóstico dado aos softwares avaliados. A partir das informações à esquerda da Tabela 2, buscou-se investigar se os alunos consideram o PAQS elaborado bom o suficiente para ser adotado em disciplinas de outros semestres. Todos $(100 \%)$ acreditam que sim, mas a maioria $(70.6 \%)$ sugere que modificações ainda precisariam ser feitas. Com as informações presentes à direita da Tabela 2, buscou-se identificar como os alunos gostariam que o diagnóstico elaborado fosse utilizado dentro do contextos das disciplina envolvidas. A maioria 
(94.1\%) indica que o diagnóstico deve ser fornecido as equipes de desenvolvimento para que melhorias possam ser implementadas ao longo da aplicação do PDS. Além disso, 35.3\% gostariam que as notas dos alunos de ESA considerassem a avaliação de qualidade feita para os produtos de software desenvolvidos.

Tabela 2. Resultados da avaliação do PAQS e diagnóstico do produto avaliado

\begin{tabular}{|l|l|l|l|l|}
$\begin{array}{l}\text { O seu plano de avaliação de qualidade } \\
\text { de software deve ser adotado como } \\
\text { padrão para a próxima disciplina? }\end{array}$ & \multicolumn{2}{l}{$\begin{array}{l}\text { Sobre o diagnóstico gerado para o software } \\
\text { que você avaliou, você gostaria que... }\end{array}$} \\
\hline Sim, ele ficou bem completo. & $\mathbf{2 9 . 4}$ & $\begin{array}{l}\text { Os desenvolvedores pudessem ler entender } \\
\text { onde existiram problemas que influenciaram } \\
\text { na qualidade do produto. }\end{array}$ & $\mathbf{9 4 . 1}$ \\
\hline $\begin{array}{l}\text { Sim, mas ele precisaria ser adaptado para } \\
\text { ser mais abrangente. }\end{array}$ & $\mathbf{7 0 . 6}$ & $\begin{array}{l}\text { A professora da disciplina de ESA } \\
\text { considerasse esta avaliação na nota dos } \\
\text { alunos desenvolvedores. }\end{array}$ & $\mathbf{3 5 . 3}$ \\
\hline $\begin{array}{l}\text { Não, ele precisa de muitas modificações } \\
\text { para se tornar um modelo para outros } \\
\text { alunos. }\end{array}$ & $\mathbf{0}$ & $\begin{array}{l}\text { Apenas a professora de GQS tivesse acesso } \\
\text { ao diagnóstico e este ficasse restrito a esta } \\
\text { disciplina. }\end{array}$ & $\mathbf{1 1 . 8}$ \\
\hline
\end{tabular}

A postura ativa no processo de ensino-aprendizagem, onde o aluno se sente motivado a auxiliar outros alunos na realização de projetos, favorece uma formação não apenas teórica, mas também humanística - os alunos precisam ser críticos, estabelecer boa comunicação, além de ter uma postura profissional ao lidar com alunos de outras turmas, nos papéis estabelecidos. Isto pode ser visto como parte da formação profissional, antecipada no ambiente universitário, contribuindo para que se qualifiquem melhor enquanto engenheiros na indústria de software.

\subsection{Avaliação da execução do projeto interdisciplinar (GQS e ESA)}

Na Tabela 3, tem-se uma visão geral sobre a opinião dos alunos com relação à execução de um projeto prático e interdisciplinar. A partir das informações à esquerda da Tabela 3 , buscou-se investigar a satisfação dos alunos no tocante a realização de um projeto prático. Como resposta, é possível observar que em sua grande maioria (93.8\%), os alunos aprovaram o método aplicado e acharam que o conteúdo foi mais facilmente apreendido. Alguns depoimentos servem para reforçar esta interpretação: "Prática é tudo. Chega de tanta teoria, a prática é fundamental e indispensável...Ter certeza que a teoria aprendida foi bem aplicada na prática" e "Já está na hora de outras disciplinas se tornarem tão práticas como foi GQS.".

As informações disponíveis à direita da Tabela 3, são direcionadas a investigar se os alunos se sentiram à vontade ao avaliar os projetos desenvolvidos na disciplina de ESA. A maior das respostas $(68.8 \%)$ demonstra satisfação dos alunos na avlaiação de produtos desenvolvidos pelos colegas, com entendimento de colaborar com feedback para melhoria do projeto em desenvolvimento. No entanto, houveram aqueles que preferiram um produto mais estável, desenvolvido por um equipe mais experiente, disponíveis no mercado (18.8\%) ou resultantes de projetos de disciplinas localizadas no final do curso (sugestão fornecida em campo aberto no questionário). Acreditamos que a motivação para avaliar produtos mais estáveis esta associada à qualidade dos artefatos 
gerados, assim como pela maior probabilidade do software ser finalizado - em comparação com aqueles produzidos por alunos do $5^{\circ}$ período (ESA). Considerando a imaturidade das equipes de ESA, foi difícil para algumas equipes GQS aplicar a teorias e as técnicas. Pode ser citado como exemplo o controle de qualidade, tendo em vista que alguns dos entregáveis da disciplina de ESA estavam incompletos ou mesmo não existiram para alguma iteração específica.

Tabela 3. Resultado da avaliação do projeto da disciplina de GQS

\begin{tabular}{|l|l|l|l|l|}
\hline Avaliação do projeto da disciplina & $\mathbf{\%}$ & Avaliação do produto avaliado & $\mathbf{\%}$ \\
\hline $\begin{array}{l}\text { Eu preferia ter tido uma disciplina apenas } \\
\text { teórica }\end{array}$ & $\mathbf{0}$ & $\begin{array}{l}\text { Teria sido mais interessante avaliar um } \\
\text { produto pronto e estável (em uso no mercado) }\end{array}$ & $\mathbf{1 8 . 8}$ \\
\hline $\begin{array}{l}\text { Eu gostei de ter realizado a parte pratica, } \\
\text { pois fixa mais o conteúdo desta } \\
\text { disciplina. }\end{array}$ & $\mathbf{2 5}$ & $\begin{array}{l}\text { Eu gostaria de ter avaliado um produto } \\
\text { desenvolvido por mim, em outra disciplina ou } \\
\text { período. }\end{array}$ & $\mathbf{1 2 . 5}$ \\
\hline $\begin{array}{l}\text { A pratica permitiu fixar o conteúdo desta } \\
\text { disciplina e revisar conceitos de }\end{array}$ & $\mathbf{6 8 . 8}$ & $\begin{array}{l}\text { Eu gostei de avaliar o produto de outros } \\
\text { alunos, e dar um feedback para que melhorem } \\
\text { o projeto. }\end{array}$ & $\mathbf{6 8 . 8}$ \\
\hline $\begin{array}{l}\text { Engenharia de Software. } \\
\text { Deu muito trabalho fazer o projeto, e nem } \\
\text { acho que aprendi muito. }\end{array}$ & $\mathbf{6 . 2}$ & $\begin{array}{l}\text { Eu gostei de avaliar o produto de outros } \\
\text { colegas, mas preferia não saber quem são os } \\
\text { desenvolvedores. }\end{array}$ & $\mathbf{0}$ \\
\hline
\end{tabular}

\section{Lições Aprendidas e Considerações Finais}

Este artigo apresentou um relato de experiência do ensino da disciplina de Gestão de Qualidade de Software integrada com a disciplina de Engenharia de Software Aplicada. A dinâmica estabelecida permitiu que os alunos de GQS avaliassem os artefatos e software produzidos por alunos da disciplina de ESA. A experiência realizada permitiu estabelecer um elo de ligação entre as disciplinas. No entanto, houve uma ênfase unidirecional, com o uso dos artefatos de ESA pelos alunos de GQS. A dinâmica do experimento, reforçada pelo relato dos alunos de GQS, destacou a importância de tornar esse elo bidirecional, com uma integração mais forte entre as duas disciplinas. Um questionário foi aplicado ao final do semestre com os alunos de GQS para sondar a satisfação destes em relação à abordagem prática e interdisciplinar aplicada na disciplina. A crítica mais recorrente dos alunos refere-se, justamente, a importância de intensificar atividades conjuntas entre os alunos de GQS e ESA a fim de permitir que os alunos de GQS participassem das apresentações dos entregáveis dos alunos de ESA desde a primeira entrega, e vice-versa. Desta forma, análises iniciais poderiam ser feitas pela equipe de qualidade e ajustes poderiam ser recomendados às equipes de desenvolvimento. Esta estratégia não foi possível nesta experiência por dois motivos: (i) a incompatibilidade de horários para reunir os alunos de ambas as turmas. No entanto, para os próximos semestres, pode-se solicitar ao departamento que as disciplinas sejam oferecidas no mesmo horário, permitindo "aulões integrados"; (ii) o fato da disciplina de ESA ser prática, cujos conceitos teóricos já foram vistos em disciplinas anteriores, enquanto que para a disciplina de GQS os conceitos teóricos e práticos foram alternados dentro do escopo de um único semestre letivo - o que leva à ritmos diferentes na condução das disciplinas. Uma estratégia de alinhamento entre as atividades das 
disciplinas precisa ser traçada para acomodar a diferença de ritmos sobre os conteúdos abordados. Este ciclo motivacional pode favorecer a construção de softwares cada vez melhores dentro do contexto das disciplinas de Engenharia de Software Aplicada.

Embora a experiência de interdisciplinaridade tenha sido bem avaliada pelos alunos, a indicação de desejo de analisar a qualidade de softwares mais estáveis, chama a atenção para uma questão relevante. Iniciativas de aproximação entre o mercado de software (indústria) e a universidade podem acontecer dentro do escopo de uma disciplina como GQS. A utilização de produtos mais estáveis do mercado pode ser uma alternativa de produtos a serem avaliados pelas equipes de qualidade formadas na disciplina. Neste sentido, as empresas podem contribuir ao permitirem associar o ensino teórico de GQS à análise de qualidade de um produto em desenvolvimento no mercado, por uma empresa específica - estabelecendo parcerias com a universidade. Assim, os dois lados ganham, tanto a universidade que fornece ao seu aluno uma vivência prática e mais eficaz no processo de ensino-aprendizagem de disciplinas relacionadas à ES, quanto a empresa, que com esta parceria pode diminuir a curva de aprendizagem e a falta de experiência prática de um novo engenheiro de software quando ele se tornar egresso da universidade e buscar o mercado de trabalho.

Adicionalmente, é importante ressaltar que alguns conteúdos mais complexos e abstratos precisam ser melhor explorados em sala de aula, a exemplo das métricas de software, assim como mais tempo deve ser dedicada à realização dos roteiros práticos correspondentes. Por fim, percebeu-se que os alunos de ESA terem conhecimento de que alunos de GQS iriam avaliar os produtos de software gerados por eles, foi um estímulo para que adotassem uma postura mais séria sobre o projeto da disciplina. $\mathrm{O}$ mesmo foi percebido com os alunos de GQS, que demonstram-se motivados tanto por avaliar um software real, quando por sentirem-se úteis dentro do contexto da disciplina de ESA.

\section{Referências}

Gnatz, M. and Kof, L. and Prilmeier, F. and Seifert, T. (2003). A Practical Approach of Teaching Software Engineering. 16th Conference on Software Engineering Education and Training (pp. 120 - 128). IEEE.

Suryn, W. (2003). Thoughts on Teaching Software Quality Engineering. 8th Annual INSPIRE Conference. April: INSPIRE.

Thompson, J., and Edwards, H. (2000). How to Teach Practical Software Quality Assurance An Experience Report. First Asia-Pacific Conference on Quality Software (pp. 181 - 187). Hong Kong: IEEE.

Wermelinger, M. and Hall, J. and Rapanotti, L. and Barroca, L. and Ramage, M. and Bandara, A. (2015). Teaching software systems thinking at The Open University. Joint Software Engineering Education and Training Conference (pp. 18-22). Florence: IEEE.

Basili, V. R. and Weiss, D. M. (1984). A Methodology for Collecting Valid Software Engineering Data. IEEE Trans. Software Eng., [S.1.], v.10, n.6, p.728-738, 1984. 